\title{
Search for multiple targets: Evidence for memory-based control of attention
}

\author{
YUJI TAKEDA \\ National Institute of Advanced Industrial Science and Technology, Tsukuba, Ibaraki, Japan
}

\begin{abstract}
There are two opposing models with regard to the function of memory in visual search: a memorydriven model and a memory-free model. Recently, Horowitz and Wolfe (2001) investigated a multipletarget search task. Participants were required to decide whether or not there were at least $n$ targets present. They demonstrated that the reaction time $\times n$ function has a positive and accelerated curve. They argued that the memory-free model predicts this curve, whereas the memory-driven model predicts a linear function. In this study, I varied the total set sizes of a multiple-target search task and fitted the models separately for each $n$ condition. The model fit indicated that the memory-driven model is more appropriate than the memory-free model in each $n$ condition. These results suggest that an amnesic process does not cause the positive acceleratedcurve of the reaction time $\times n$ function but that it is the result of the time needed to examine each additional $n$ item.
\end{abstract}

Recently, researchers have investigated whether the serial deployment of attention in the visual search process is controlled by a memory-driven mechanism or by a memory-free mechanism. In the memory-driven model (see Klein, 2000, for a review), it is assumed that previously attended distractors are memorized and inhibited to prevent the ineffective reexamination of rejected distractors during the subsequent search. Conversely, in the memory-free model (e.g., Horowitz \& Wolfe, 1998), it is assumed that no record is kept on the deployment of attention during a search. The important difference between the two models is whether or not previously checked and rejected distractors are reexamined.

In previous studies, the function of memory in visual search tasks has been investigated by using three methods. These are by (1) using reaction times (RTs) for the detecting of probes among distractors (Klein, 1988; Müller \& von Mühlenen, 2000; Ogawa, Takeda, \& Yagi, 2002; Takeda \& Yagi, 2000), (2) using the refixation frequency of eye movements (Gilchrist \& Harvey, 2000; Peterson, Kramer, Wang, Irwin, \& McCarley, 2001), and (3) using the slope of the search function in a dynamic display (Horowitz \& Wolfe, 1998; Kristjánsson, 2000). Studies in which the first method has been used have consistently shown increased RTs for the detecting of probes at the locations of previously attended distractors, suggesting that these distractors are inhibited. Although this finding appears to support the memory-driven model, it has been argued that RTs for the detecting of probes may not be a di-

I thank C. M. Moore, M. S. Peterson, and T. S. Horowitz for helpful comments on an earlier version of this article. Correspondence concerning this article should be addressed to Y. Takeda, Visual Cognition Group, Institute for Human Science and Biomedical Engineering, National Institute of Advanced Industrial Science and Technology (AIST), Tsukuba Central 6, 1-1-1 Higashi, Tsukuba, 305-8566 Ibaraki, Japan (e-mail: yuji-takeda@aist.go.jp). rect measure of the control of attention during a search task. On the contrary, studies in which the second and third methods have been used have reported inconsistent findings. Using the second method, Gilchrist and Harvey demonstrated frequent refixation to previously fixated distractors, whereas Peterson et al. found few refixations during a search. Using the third method, Horowitz and Wolfe (1998) found no difference in the slopes of static and dynamic displays (search items were randomly relocated every $110 \mathrm{msec}$ ). However, Kristjánsson reported that the slope of a dynamic display was significantly steeper than that of a static display when large set sizes were used (but see Horowitz \& Wolfe, 2003).

Horowitz and Wolfe (2001) have proposed a clever method of investigating the function of memory in visual search tasks. In their study, participants were required to respond whether or not there were at least $n$ targets present. Horowitz and Wolfe (2001) argued that the memory-free model predicts different functions from the memorydriven model concerning variations in the number of search targets $(n)$, the number of actual targets in the display $(t)$, and the number of distractors in the display $(d)$. In the pure memory-driven model (i.e., previously checked items are memorized perfectly), the expected value $(E)$ of the number of checked items before $n$ targets are detected is given by the following equation:

$$
E=\frac{n(t+d+1)}{t+1}
$$

On the other hand, in the memory-free model, the expected value $(E)$ is expressed by the following equation:

$$
E=\sum_{i=1}^{n}\left[1+\left(\frac{d+i-1}{t-i+1}\right)\right]
$$

If we assume that the number of actual targets $(t)$ and distractors $(d)$ in the display are constant, in the memorydriven model, the number of search targets $(n)$ would affect 
the expected value $(E)$ linearly, whereas this would not be the case in the memory-free model. The memory-free model predicts an accelerating increase in RTs, because it will take more samples to stumble across an unexamined item. Horowitz and Wolfe (2001) demonstrated that the RT $\times n$ function under a constant set size is positive and accelerating, rather than linear (the total number of targets and distractors was 16). On the basis of this finding, they concluded that visual search has no memory.

Although the results from multiple-target searches appear to provide strong evidence supporting the memoryfree model, this evidence is not as clear as it seems. The conclusion of Horowitz and Wolfe's (2001) study was based on the assumption that the number of search targets $(n)$ is irrelevant to the search rate (i.e., the time for examining each item). This assumption is questionable, because the mental load is heavier when participants look for a large number of targets than when they look for a small number of targets. Recent studies have demonstrated that spatial working memory load and executive processes during the visual search slows the search process (Han \& Kim, 2002; Oh \& Kim, 2002), although Woodman, Vogel, and Luck (2001) proposed that the storing of features of objects did not affect search efficiency. In the multiple-target search task, during visual search, participants were required to explicitly enumerate the examined targets and memorize their locations in order to prevent recounting of previously attended targets. These processes are based on working memory (Tuholski, Engle, \& Baylis, 2001). Therefore, executive process load (i.e., enumeration) and spatial working memory load (i.e., explicit memory for the examined targets) are heavier in a large-number search condition (e.g., when participants look for five targets) than in a smallnumber search condition (e.g., when participants look for two targets), resulting in a longer search rate in the largenumber search condition than in the small-number search condition. If the search rate increases with increasing numbers of search targets $(n)$, the RT $\times n$ function would accelerate positively, even if previously checked items are perfectly memorized and inhibited.

In the present study, using the multiple-target search paradigm, I varied the total set size and the number of actual targets in the display and examined the RT function in these two conditions when the numbers of search targets (n) were constant. The memory-driven model and the memory-free model predict different RT functions for the interaction between total set size $(t+d)$ and the number of actual targets in the display $(t)$, even when the number of search targets $(n)$ is constant. Thus, the present study was designed to isolate the confounding effects of mental load and clarify the function of memory in visual search.

\section{METHOD}

\section{Participants}

Forty-two graduate and undergraduate students (10 males and 32 females, 18-27 years of age, $M=21.2$ years), who were paid vol- unteers, with normal or corrected-to-normal vision, participated in the study.

\section{Apparatus and Stimuli}

Stimuli were displayed on a 17 -in. color monitor (IIYAMA A702H, with 1,280 $\times 1,024$ pixel resolution) controlled by an IBM PC AT-compatible computer (Dell Precision 620). All the stimuli were presented on a dark background in a semi-dark room. The participants observed the stimuli at a $58-\mathrm{cm}$ viewing distance.

Each trial began with the presentation of a white fixation cross ("+", $0.25^{\circ} \times 0.25^{\circ}$ ) at the center of the display, which remained until the end of the trial. After 1,000 msec from the onset of the fixation, a search stimulus appeared at randomly chosen locations defined by the intersection of an invisible grid of $6 \times 6$ lines $\left(6^{\circ} \times 6^{\circ}\right)$. The search stimuli consisted of white circles (targets) and white arcs (distractors). The diameters of these items subtended $0.6^{\circ}$ of visual angle. One eighth of the circle was cut to form a gap to constitute each arc. The location of the gap was chosen at random. Set sizes of 12,18 , and 24 were used with equal probability. The search stimulus was displayed until the participant responded.

\section{Design and Procedure}

There were three conditions related to the number of search targets: a one-target condition, a three-target condition, and a five-target condition. These conditions were tested between participants (14 participants for each condition). Before the experiment, the participants were instructed on how many targets (circles) to search for ( $n$ ) and were required to respond whether or not at least $n$ targets were present on the display by pressing one of two keys with their right or left index fingers. Immediate auditory error feedback was provided. In half of the trials, $n-1$ targets were displayed (i.e., negative trials). In the remaining trials, $n$ targets, $n+1$ targets, $n+2$ targets, $n+3$ targets, or $n+4$ targets were presented with equal probability (i.e., positive trials). Each participant performed 1 practice block and 18 experimental blocks. Each block consisted of 60 trials.

\section{RESULTS AND DISCUSSION}

The data of 1 participant in the five-target search condition were excluded from further analysis because she did not understand the task correctly. The mean correct RTs and mean error rates are shown in Figure 1. For each condition, the RTs in positive trials were subjected to a two-way analysis of variance (ANOVA) with the number of actual targets $(n, n+1, n+2, n+3$, or $n+4)$ and set sizes $(12,18$, or 24$)$ as the main terms. In the one-target search condition, the main effects of the number of actual targets $[F(4,52)=48.4, p<.0001]$ and set size $[F(2,26)=$ $19.9, p<.0001]$ were significant. In the three-target search condition, the main effects of the number of actual targets $[F(4,52)=105.7, p<.0001]$ and set size $[F(2,26)=160.0, p<.0001]$ were significant. There was also a significant interaction between them $[F(8,104)=$ $7.2, p<.0001]$. In the five-target search condition, the main effects of the number of actual targets $[F(4,48)=$ $85.2, p<.0001]$ and set size $[F(2,24)=90.0, p<.0001]$ were significant. There was also a significant interaction between them $[F(8,96)=4.2, p<.0005]$.

Error rates were also subjected to a two-way ANOVA with the same factors as those in the RT analysis. In the one-target search condition, the main effects of the number of actual targets $[F(4,52)=147.3, p<.0001]$ and set size 

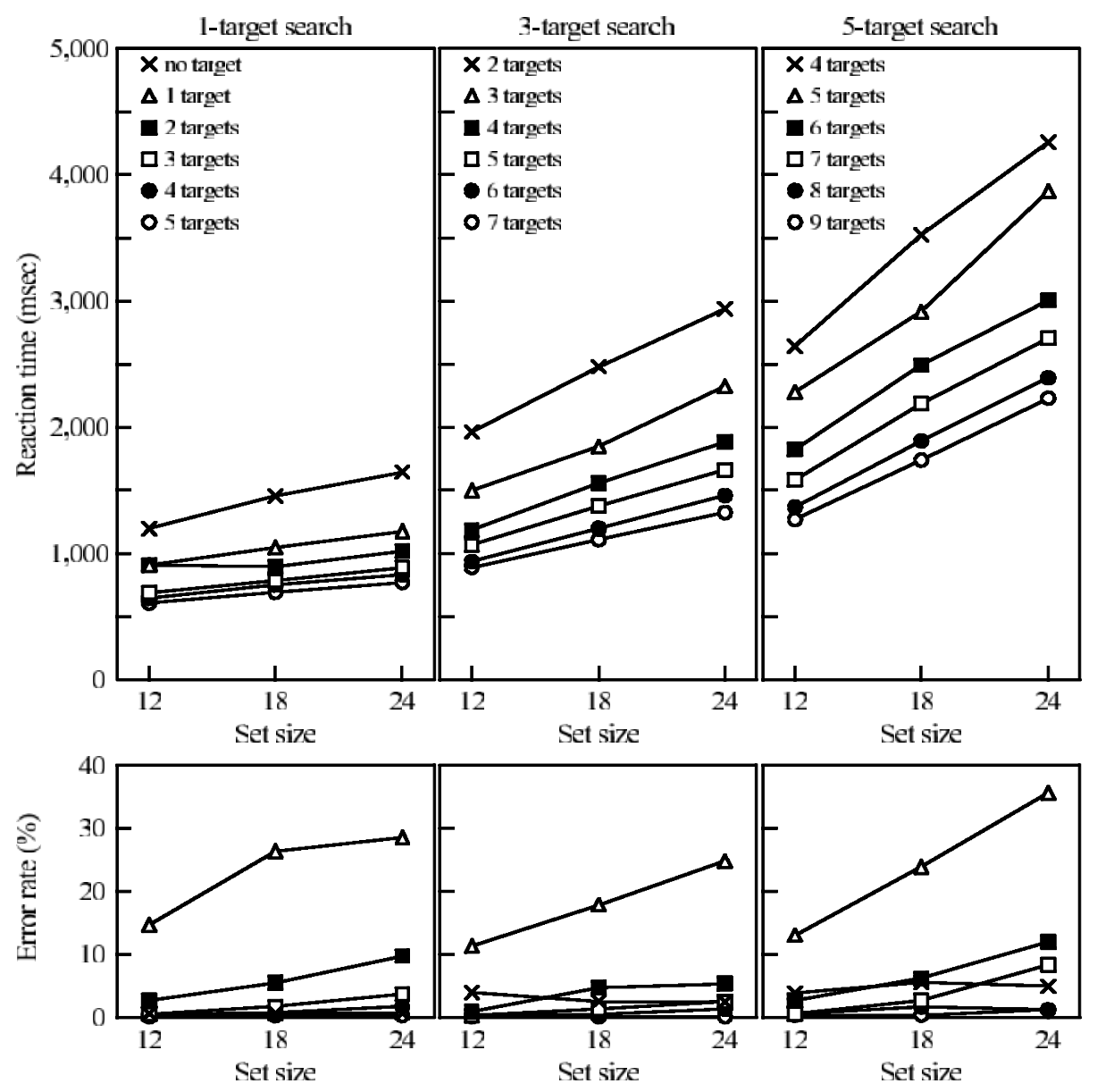

Figure 1. Mean correct reaction times and error rates.

$[F(2,26)=39.0, p<.0001]$ were significant. There was also a significant interaction between them $[F(8,104)=$ $7.1, p<.0001]$. In the three-target search condition, the main effects of the number of actual targets $[F(4,52)=$ $123.1, p<.0001]$ and set size $[F(2,26)=18.2, p<$ .0001] were significant. There was also a significant interaction between them $[F(8,104)=5.4, p<.0001]$. In the five-target search condition, the main effects of the number of actual targets $[F(4,48)=49.9, p<.0001]$ and set size $[F(2,24)=22.8, p<.0001]$ were significant. There was also a significant interaction between them $[F(8,96)=9.1, p<.0001]$. RTs and error rates increased with increasing set sizes and with decreasing numbers of actual targets. The slope of the RT $\times$ set size function increased with decreasing numbers of actual targets, although it was not significant in the one-target condition.

Horowitz and Wolfe (2001) examined the RT $\times n$ function with the same number of actual targets and demonstrated a positive accelerated curve. Figure 2 shows the RT $\times n$ function in the trials with five actual targets. As in Horowitz and Wolfe (2001), the curves were positively accelerated in each set size. The RTs were subjected to a quadratic trend analysis separately for each set size. In all set sizes, the quadratic trends were significant $[F(1,38)=13.8, p<.001$, in set size $12 ; F(1,38)=$ $9.0, p<.005$, in set size 18 ; and $F(1,38)=6.8, p<.05$, in set size 24], suggesting that the curves were positively accelerated. This result is consistent with that of Horowitz

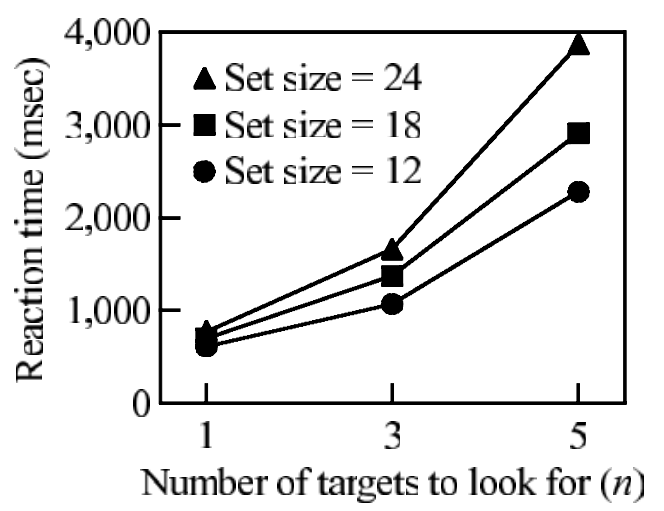

Figure 2. Mean correct reaction times in the trials in which five actual targets were presented. 
and Wolfe (2001). However, as I have mentioned in the introduction, it is possible that these accelerated curves were not a result of the reexamining of rejected distractors but were the result of an increased search rate caused by increasing numbers of search targets $(n)$. This possibility was examined in a model-fitting analysis conducted separately for each $n$.

\section{Model Fitting}

To examine whether attention was controlled by a memory-driven mechanism or, alternatively, by a memoryfree mechanism, the RTs from the positive trials were fitted to the models. RT in the memory-driven model can be predicted with the following equation:

$$
\mathrm{RT}=b+\frac{n r(t+d+1)}{t+1} .
$$

RT in the memory-free model can be predicted with the following equation:

$$
\mathrm{RT}=b+\sum_{i=1}^{n}\left[1+\left(\frac{d+i-1}{t-i+1}\right)\right] r .
$$

Equations 3 and 4 were constructed by adding two additional parameters to Equations 1 and 2 (i.e., an intercept, $b$, and a search rate, $r$ ) to account for the expected value of the number of items to be checked before $n$ targets $(E)$ are detected (see the introduction). In both the memorydriven and the memory-free models, the equations indicate that RT should increase linearly with the expected value of checked items $(E)$, even though the memorydriven model and the memory-free model predict different $E$ values. Figure 3 shows the RT $\times E$ value functions separately for each number of search targets $(n)$. The error range in each $n$ was smaller in the memory-driven model than in the memory-free model.

To examine the models statistically, each model was fitted separately for each number of search targets $(n)$, using a least root-mean squared (RMS) error criterion. The 15 data points in the positive trials (5 actual target conditions $\times$ 3 set sizes) were fitted to the models. Table 1 shows the search rate, the intercept, and the RMS error of each model. The model fitting resulted in smaller RMS errors in the memory-driven model than in the memory-free model in all $n$ conditions. This indicates that the memory-driven model is a more appropriate explanation of the function of memory in visual search than is the memory-free model.

Each model was fitted separately for each subject. A $t$ test was performed on the RMS errors for each condition of the number of search targets. The RMS error was significantly smaller in the memory-driven model than in the memory-free model in the one-target search condition $[t(13)=7.4, p<.0001]$ and in the three-target search condition $[t(13)=3.6, p<.005]$. The $t$ test failed to reach significance in the five-target search condition $[t(12)=1.8, p=.10]$.

As in Horowitz and Wolfe (2001), the curves of the RT $\times n$ function in the present study are positively accelerated in each set size (see Figure 2). It is possible that the search rate increases with increasing numbers of search targets because the mental load is heavier when participants look for a large number of targets relative to a small number of targets. In the present study, the fit in the memory-driven model assumed that the search rate increased with increasing numbers of search targets $(54.0 \mathrm{msec} / \mathrm{item}$ in the one-target search condition, $102.3 \mathrm{msec} /$ item in the three-target search condition, and $167.9 \mathrm{msec} /$ item in the five-target search condition). The search rates estimated on the memory-driven model were subjected to a one-way ANOVA with the number of search targets (one, three, or five) as the main factor (between-subjects design). The main effect was significant $[F(2,38)=26.9, p<.0001]$. This indicates that the positive and accelerated curves of the RT $\times n$ function are the result of an increasing search rate with increasing numbers of search targets and are not due to the reexamination of rejected distractors.

Model fitting in the memory-driven model was based on the assumption that previously attended items were perfectly memorized and no reexamination of those items occurred. On the other hand, the memory-free model assumes that none of the previously attended items was memorized and that any item could be examined with equal probability. The limited-capacity model lies halfway between the pure memory-driven model and the pure memory-free model. The limited-capacity model, like the memory-driven model, assumes that when the number of previously attended items is less than capacity, those items are memorized and inhibited. However, when the number of attended items is over the capacity, no more items are memorized, which is similar to the memory-free model. The limited-capacity model allows the capacity to vary between zero and infinity. If the capacity is zero, the limited-capacity model functions similarly to the memory-free model, and if the capacity is infinity (or at least more than the set size), the limited-capacity model functions similarly to the memory-driven model.

The limited-capacity model was fitted separately for each number of search targets, varying the capacity from 0 (i.e., the memory-free model) to 24 (i.e., the memorydriven model). Table 1 indicates that the capacity yielded a least RMS error. The capacity was approximately 20 items in each $n$ search condition (22 items in the onetarget search condition, 21 items in the three-target search condition, and 19 items in the five-target search condition), whereas Horowitz and Wolfe (2001) reported a smaller capacity (3 or 5 items). The estimated capacity in this study is consistent with the findings from Peterson et al.'s (2001) eye movement study, in which they found nearly perfect memory in visual search.

Although the present study demonstrated a large memory capacity in visual search, the capacity failed to reach set size (i.e., 24 items). This finding indicates a deterioration of memory during visual search. There are two possible reasons for this deterioration: time and the number of intervening items checked. The results of this study showed that the search rate $(r)$ in the five-target search condition was three times longer than that in the one-target search condition under the limited-capacity 

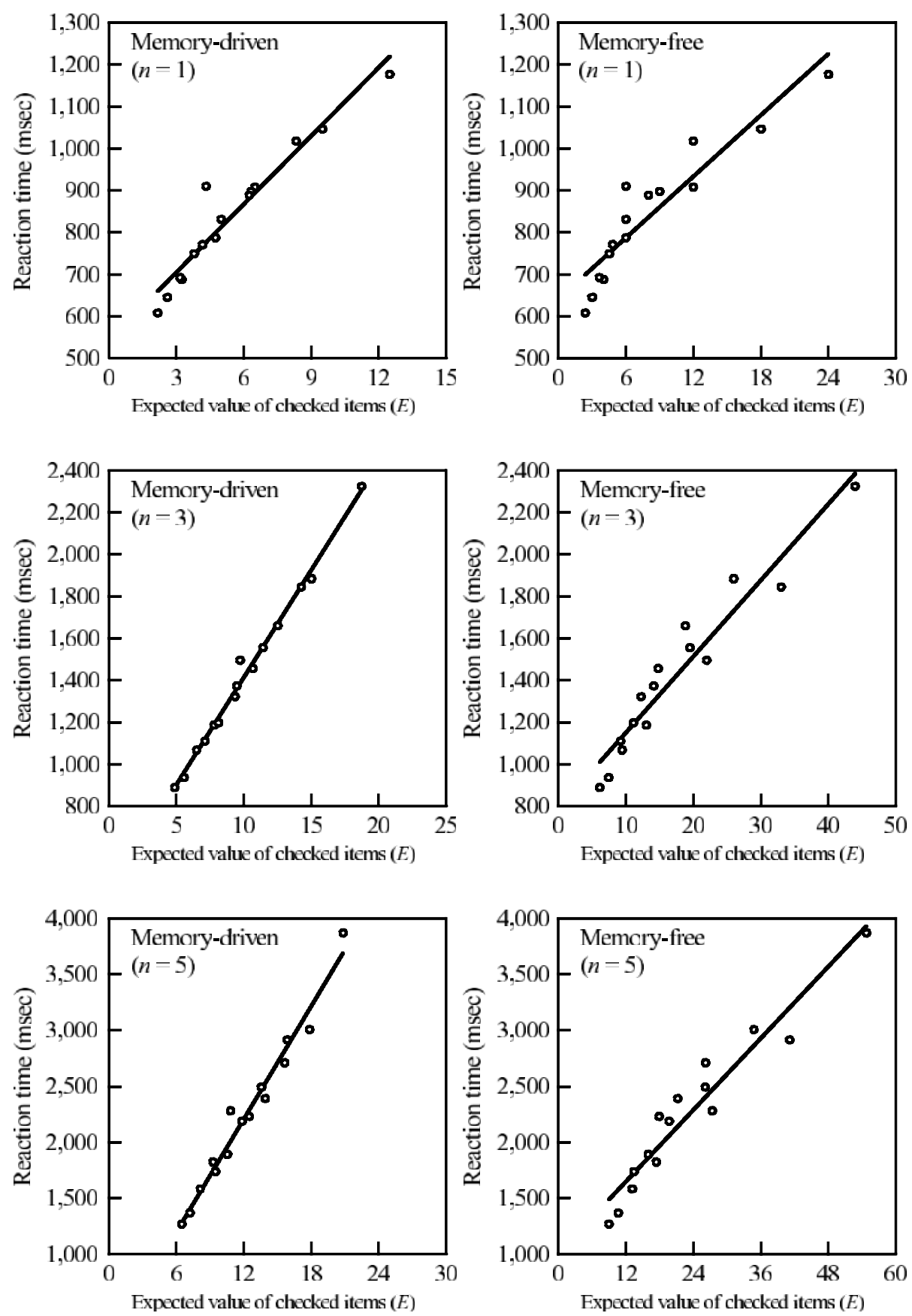

Figure 3. The functions of the RT $\times E$ value separately for each number of search targets $(n)$.

model fitting. If memory decays with time, the estimated capacity of memory in the five-target search condition should be one third of that in the one-target search condition. This study, however, demonstrated few differences in capacity between the one-target search condition and the five-target search condition. Therefore, we suggest that the number of intervening items checked, rather than time, is the main cause of memory decay.
Previous studies in which the probing paradigm have been used have indicated that the memory capacity in visual search is over 8 items (Ogawa et al., 2002; Takeda $\&$ Yagi, 2000). They argued that memory capacity in visual search is more than FINST (Pylyshyn \& Storm, 1988) and more than visual short-term memory in change detection tasks (e.g., Luck \& Vogel, 1997). The fit in the limited-capacity model in this study (approximately 20 
Table 1

Results of Model Fitting

\begin{tabular}{|c|c|c|c|c|}
\hline Model & $\begin{array}{l}\text { Search Rate } \\
(\mathrm{msec} / \mathrm{item})\end{array}$ & $\begin{array}{c}\text { Intercept } \\
(\mathrm{msec})\end{array}$ & $\begin{array}{c}\text { Capacity } \\
\text { (items) }\end{array}$ & $\begin{array}{c}\text { RMS Error } \\
(\mathrm{msec})\end{array}$ \\
\hline \multicolumn{5}{|c|}{ One-Target Search $(n=1)$} \\
\hline Memory driven & 54.0 & 543.6 & & 42.0 \\
\hline Memory free & 24.3 & 641.3 & & 57.4 \\
\hline Limited capacity & 53.7 & 544.4 & 22 & 41.9 \\
\hline \multicolumn{5}{|c|}{ Three-Target Search $(n=3)$} \\
\hline Memory driven & 102.3 & 388.5 & & 33.6 \\
\hline Memory free & 36.2 & 791.2 & & 104.3 \\
\hline Limited capacity & 101.2 & 398.5 & 21 & 31.7 \\
\hline \multicolumn{5}{|c|}{ Five-Target Search $(n=5)$} \\
\hline Memory driven & 167.9 & 189.6 & & 113.2 \\
\hline Memory free & 53.2 & $1,011.6$ & & 185.0 \\
\hline Limited capacity & 156.2 & 310.8 & 19 & 100.4 \\
\hline
\end{tabular}

items) is consistent with the results of studies in which the probing paradigm has been used. However, the results of studies of inhibition of return (IOR) with the spatialcuing paradigm are not consistent with our study. For instance, Snyder and Kingstone (2000) demonstrated that IOR decayed with time or with the number of intervening cues when six spatial cues were presented sequentially. They argued that IOR occurs at no more than five spatially separate locations. The discrepancy in capacity of inhibition may be due to procedural differences between the spatial-cuing paradigm and the visual search paradigm: The luminance increment cues guided participants' attention exogenously in the spatial-cuing paradigm, whereas participants deployed their attention to items endogenously in the visual search paradigm.

The results of this study demonstrated a memory-driven, or a large-capacity (approximately 20 items), mechanism of visual search. The functions of RTs in the multipletarget search task can provide more direct evidence regarding the nature of memory in visual search, as compared with other measures, such as RTs for probing, refixation frequency of eye movements, and $\mathrm{RT} \times$ set size function in a dynamic display. On the basis of these considerations, I conclude that during visual search, previously attended items are memorized and inhibited to prevent the ineffective reexamination of rejected distractors.

\section{REFERENCES}

Gilchrist, I. D., \& HARVEY, M. (2000). Refixation frequency and memory mechanisms in visual search. Current Biology, 10, 1209-1212.

HAN, S.-H., \& KIM, M.-S. (2002, July). Visual search does not remain efficient when working memory is working. Paper presented at the Second Asian Conference on Vision, Gyeongju, Korea.

Horowitz, T. S., \& Wolfe, J. M. (1998). Visual search has no memory. Nature, 394, 575-577.

Horowitz, T. S., \& Wolfe, J. M. (2001). Search for multiple targets: Remember the targets, forget the search. Perception \& Psychophysics, 63, 272-285.

Horowitz, T. S., \& Wolfe, J. M. (2003). Memory for rejected distractors in visual search? Visual Cognition, 10, 257-298.

KLEIN, R. M. (1988). Inhibitory tagging system facilitates visual search. Nature, 334, 430-431.

KLEIN, R. M. (2000). Inhibition of return. Trends in Cognitive Sciences, 4, 138-147.

KRISTJÁNSSON, Á. (2000). In search of remembrance: Evidence for memory in visual search. Psychological Science, 11, 328-332.

LUCK, S. J., \& VOGEL, E. K. (1997). The capacity of visual working memory for features and conjunctions. Nature, 390, 279-291.

MülleR, H. J., \& VON MüHLENEN, A. (2000). Probing distractor inhibition in visual search: Inhibition of return. Journal of Experimental Psychology: Human Perception \& Performance, 26, 1591-1605.

OgaWA, H., TAKedA, Y., \& YAGI, A. (2002). Inhibitory tagging on randomly moving objects. Psychological Science, 13, 125-129.

OH, S.-H., \& KIM, M.-S. (2002, July). Visual search efficiency is affected by spatial working memory. Paper presented at the Second Asian Conference on Vision, Gyeongju, Korea.

Peterson, M. S., Kramer, A. F., Wang, R. F., Irwin, D. E., \& MCCARLEY, J. S. (2001). Visual search has memory. Psychological Science, 12, 287-292.

Pylyshyn, Z. W., \& STORM, R. W. (1988). Tracking multiple independent targets: Evidence for a parallel tracking mechanism. Spatial Vision, 3, 179-197.

SNYdER, J. J., \& KingSTONe, A. (2000). Inhibition of return and visual search: How many separate loci are inhibited? Perception \& Psychophysics, 62, 452-458.

TAKEDA, Y., \& YAGI, A. (2000). Inhibitory tagging in visual search can be found if search stimuli remain visible. Perception \& Psychophysics, 62, 927-934.

Tuholski, S. W., ENGLe, R. W., \& BAYlis, G. C. (2001). Individualdifferences in working memory capacity and enumeration. Memory \& Cognition, 29, 484-492.

Woodman, G. F., Voge L, E. K., \& LUCK, S. J. (2001). Visual search remains efficient when visual working memory is full. Psychological Science, 12, 219-224.

(Manuscript received April 22, 2002; revision accepted for publication December 18, 2002.) 